\title{
Spin-orbit interaction in chiral carbon nanotubes probed in pulsed magnetic fields
}

\author{
S. H. Jhang, ${ }^{1}$ M. Marganska, ${ }^{2}$ Y. Skourski ${ }^{3}$ D. Preusche, ${ }^{1}$ B. Witkamp, ${ }^{4}$ M. Grifoni, ${ }^{2}$ H. van der Zant, ${ }^{4}$ \\ J. Wosnitza, ${ }^{3}$ and C. Strunk ${ }^{1, *}$ \\ ${ }^{1}$ Institute of Experimental and Applied Physics, University of Regensburg, 93040 Regensburg, Germany \\ ${ }^{2}$ Institute for Theoretical Physics, University of Regensburg, 93040 Regensburg, Germany \\ ${ }^{3}$ Dresden High Magnetic Field Laboratory, Forschungszentrum Dresden-Rossendorf, 01314 Dresden, Germany \\ ${ }^{4}$ Kavli Institute of Nanoscience, Delft University of Technology, 2628 CJ Delft, The Netherlands
}

(Received 17 June 2010; published 15 July 2010)

\begin{abstract}
The magnetoconductance of an open carbon nanotube (CNT)-quantum wire was measured in pulsed magnetic fields. At low temperatures, we find a peculiar split magnetoconductance peak close to the chargeneutrality point. Our analysis of the data reveals that this splitting is intimately connected to the spin-orbit interaction and the tube chirality. Band-structure calculations suggest that the current in the peak regions is highly spin polarized, which calls for application in future CNT-based spintronic devices.
\end{abstract}

DOI: 10.1103/PhysRevB.82.041404

PACS number(s): 73.63.Fg, 75.47.- m, 73.23.Ad, 85.75.- -d

An efficient source of spin-polarized electrons is one of the important building blocks of a future spin-based electronics. ${ }^{1}$ Very high degrees of polarization can potentially be achieved by exploiting spin-orbit interaction (SOI). ${ }^{2,3}$ Based on the low atomic number $Z=6$ of carbon, the spin-orbit interaction in carbon nanotubes (CNTs) was mostly believed to be very weak, until a recent experiment ${ }^{4}$ has demonstrated the effect of spin-orbit interaction in clean CNT quantum dots.

In this Rapid Communication, we present magnetoconductance (MC) data for the complementary situation of an open CNT-quantum wire obtained in pulsed magnetic fields. Open quantum wires allow much higher currents (up to microampere) since a whole band participates in the transport rather than the individual levels in the quantum-dot regime. In a parallel magnetic field $B_{\|}$, a small band-gap CNT evolves via a metallic state into a semiconducting one, resulting in a typical peak in the MC. ${ }^{5,6}$ In one of our tubes, however, we observed a splitting of this MC peak into two peaks at low temperature. Recording MC traces at different gate voltage $V_{g}$ shows that the splitting vanishes when moving away from the charge-neutrality point (CNP). A thorough comparison to band-structure calculations reveals that the splitting is explained by the SOI, which becomes strong for small tube diameters. Our analysis predicts a highly spinpolarized current in the peak regions.

The experiments have been performed on devices made of individual CNTs prepared on $\mathrm{Si} / \mathrm{SiO}_{2} / \mathrm{Si}_{3} \mathrm{~N}_{4}$ substrates. The heavily $p$-doped $\mathrm{Si}$ was used as a backgate and the thickness of the insulating layer was $350 \mathrm{~nm}$. CNTs were grown by means of a chemical vapor deposition method ${ }^{7}$ and $\operatorname{Pd}(50$ $\mathrm{nm})$ electrodes were defined on top of the tubes by e-beam lithography. In order to exclude strain effects on the band structure, ${ }^{8}$ only straight and long $(\sim 50 \mu \mathrm{m})$ CNTs were selected for devices and the distance between two Pd electrodes was $\sim 500 \mathrm{~nm}$. The dc magnetoconductance was studied in pulsed magnetic fields of up to $60 \mathrm{~T}$, applied parallel to the tube axis. The accuracy of the alignment was $\sim \pm 5^{\circ}$ (see supplementary material for further experimental details). ${ }^{9}$

Figure 1(a) shows the magnetoconductance $G\left(B_{\|}\right)$of a small-band-gap CNT device located near the CNP (diameter $d \sim 1.5 \mathrm{~nm}$ ). At $82 \mathrm{~K}$, the conductance $G$ of the tube initially increases to reach a maximum at $B_{0}=5.9 \mathrm{~T}$, then it exponentially drops to zero at higher fields due to the AharonovBohm (AB) effect. ${ }^{5,10}$ Interestingly, when the device was
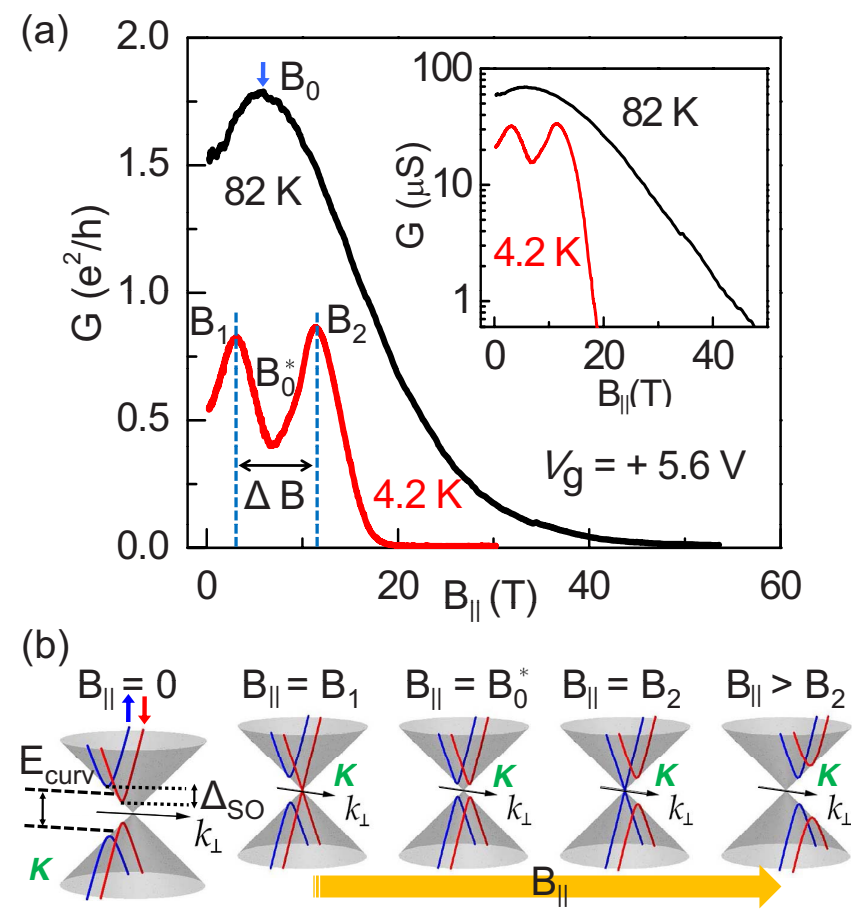

FIG. 1. (Color online) (a) MC of a small-band-gap CNT device near the CNP measured at 82 and $4.2 \mathrm{~K}$. The inset shows the MC in a semilog scale. The observed double peak in $G\left(B_{\|}\right)$defines the characteristic fields $B_{0}^{*}, B_{1}$, and $B_{2}$. (b) One of the Dirac cones near the $K$ points, intersected by lines of allowed $k_{\perp}$ values for a smallband-gap CNT with SOI. Spin-up and spin-down bands (marked by arrows) display a curvature-induced band gap $E_{\text {curv }}$ and are separated by $\Delta_{\text {SO }}$, due to the SOI. With increasing $B_{\|}$, spin-split subbands shift due to the $\mathrm{AB}$ effect and cross the $K\left(K^{\prime}\right)$ point, successively closing the energy gap at $B_{1}$ and $B_{2}$. For simplicity, the Zeeman term and $\varepsilon_{\text {SO }}$, an additional Zeeman-type term induced by the SOI, are neglected. However, $B_{1}$ and $B_{2}$ are not affected by those terms (see supplementary material) (Ref. 9). 
cooled down to $4.2 \mathrm{~K}$, the conductance maximum $G_{\max }$ at $B_{0}$ was split into two distinct peaks at magnetic fields $B_{1}$ $=3.1 \mathrm{~T}$ and $B_{2}=11.1 \mathrm{~T}$. We note that these peaks are symmetric with respect to the conductance dip at $B_{0}^{*} \approx 7 \mathrm{~T}$, and $G\left(B_{0}^{*}\right)$ is similar in magnitude to $G\left(B_{\|}=0\right)$. The key to the explanation of the data lies in the magnetic field dependence of the one-dimensional band structure.

A specific CNT is uniquely labeled by the chiral indices $(n, m)$, which define the chiral angle $\theta$ and the quantized values of the transversal wave vector $k_{\perp} \cdot{ }^{11}$ The values of $k_{\perp}$, combined with the graphene dispersion cones, determine the quasi-one-dimensional band structure of the CNTs. A given CNT is metallic if the lines of allowed $k_{\perp}$ cross the Dirac points $K$ and $K^{\prime}$; otherwise it is semiconducting. For nominally metallic CNTs $(n-m=3 l$, with $l$ an integer), the dispersion relation $E\left(k_{\|}\right)$near the Dirac points reads ${ }^{4,12-15}$

$$
\begin{gathered}
E\left(k_{\|}\right)= \pm \hbar v_{F} \sqrt{k_{\|}^{2}+k_{\perp}^{2}}+\left(\frac{g}{2} \mu_{B} B_{\|}+\tau \varepsilon_{\mathrm{SO}}\right) \sigma, \\
k_{\perp}=k_{\mathrm{AB}}+k_{\perp}^{0}+k_{\mathrm{SO}},
\end{gathered}
$$

where $k_{\|}$is the wave vector parallel to the tube axis, $v_{F}$ the Fermi velocity, $\frac{g}{2} \mu_{B} B_{\|} \sigma$ being the Zeeman term with $\sigma$ $= \pm 1$ for spin parallel/antiparallel to the tube axis, and $\tau$ $= \pm 1$ for the $K$ and $K^{\prime}$ Dirac points. The transversal wave vector $k_{\perp}$ contains three distinct contributions, which are discussed below.

The Aharonov-Bohm flux $\phi_{\mathrm{AB}}=B_{\|} \pi d^{2} / 4$ results in a shift $k_{\mathrm{AB}}=(2 / d)\left(\phi_{\mathrm{AB}} / \phi_{0}\right)$ of $k_{\perp}$, where $\phi_{0}=h / e$ is the flux quantum. Therefore, one can convert a metallic CNT into a semiconducting one, or vice versa, by tuning the allowed values of $k_{\perp}$ with a magnetic field parallel to the tube axis. ${ }^{5,10,16-18}$

In addition, curvature ${ }^{19}$ affects the allowed values of $k_{\perp}$ and induces small band gaps in nominally metallic CNTs. The curvature-induced shift ${ }^{19,20} k_{\perp}^{0}=-\tau a_{0} \cos (3 \theta) /(2 d)^{2}$ of the allowed $k$ states results in a band gap $E_{\text {curv }}=2 \hbar v_{F}\left|k_{\perp}^{0}\right|$ at $B_{\|}=0$, where $a_{0}$ is the $\mathrm{C}-\mathrm{C}$ bond length.

A second consequence of curvature is a spin-dependent shift,

$$
k_{\mathrm{SO}}=-\sigma(2 / d)\left(\phi_{\mathrm{SO}} / \phi_{0}\right),
$$

of $k_{\perp}$ by the spin orbit interaction ${ }^{4,12-15,21,22}$ which removes the fourfold spin and $K, K^{\prime}$ degeneracy in favor of two Kramers doublets corresponding to parallel and antiparallel alignment of orbital and spin magnetic moments. This SOIinduced shift in $k_{\perp}$ is equivalent to the presence of an $\mathrm{AB}$ flux $\phi_{\mathrm{SO}} \approx 10^{-3} \phi_{0},{ }^{4,12}$ and produces a spin-orbit energy splitting $\Delta_{\mathrm{SO}}=2 \hbar v_{F}\left|k_{\mathrm{SO}}\right|$. For a CNT with $d \sim 1 \mathrm{~nm}, \phi_{\mathrm{SO}}$ corresponds to $\simeq 5 \mathrm{~T}$ while $\phi_{0}$ is $\simeq 5000 \mathrm{~T}$.

In contrast, the term with $\varepsilon_{\mathrm{SO}}=-\delta \cos (3 \theta) / d$, added to the root in the Eq. (1) (such as the Zeeman term), solely shifts the energy but not $k_{\perp}$, leading to an asymmetric spin-orbit energy splitting for the hole $\left(\Delta_{\mathrm{SO}}+2 \varepsilon_{\mathrm{SO}}\right)$ and the electron band $\left(\Delta_{\mathrm{SO}}-2 \varepsilon_{\mathrm{SO}}\right)$ of chiral metallic tubes. ${ }^{14,15}$ As $\varepsilon_{\mathrm{SO}}$ contains the factor $\cos (3 \theta)$, it is small for near armchair tubes. The parameter $\delta$ ranges from $0.3-0.7 \mathrm{~nm} \mathrm{meV} .{ }^{14,15}$

The resulting evolution of the band structure in magnetic field is visualized in Fig. 1(b). At zero field, the band gap
$E_{g}^{0}=E_{\text {curv }}-\Delta_{\text {SO }}$ is reduced by the SOI. With the application of $B_{\|}$, the two spin subbands separated by the SOI cross the corner point of the Brillouin zone (either at $K$ or $K^{\prime}$ ), thus explaining two subsequent MC peaks at $B_{1}$ and $B_{2}$. In between, a conductance dip appears at $B_{0}^{*}$ when the spin subbands are located symmetrically around the corner point. If the Zeeman-type terms in Eq. (1) are neglected the energy gap has a local maximum at $k_{\mathrm{AB}}=-k_{\perp}^{0}$ corresponding to $E_{g}\left(B_{0}^{*}\right) \approx \Delta_{\mathrm{SO}}$. The distance between the two peaks, $\Delta B$ $=\left(4 / \pi d^{2}\right) \Delta \phi_{\mathrm{AB}}$, is determined by $\Delta \phi_{\mathrm{AB}}=2 \phi_{\mathrm{SO}}$ (the factor 2 comes from $\sigma= \pm 1$ ). For the observed values of $\Delta B=8 \mathrm{~T}$ and $d=1.5 \mathrm{~nm}$, we find

$$
\phi_{\mathrm{SO}}=\frac{\pi d^{2} \Delta B}{8} \approx 1.7 \times 10^{-3} \phi_{0} .
$$

For a conservative confidence interval of $\pm 0.5 \mathrm{~nm}$ for $d$ determined with an atomic force microscope, one obtains $0.76<10^{3} \phi_{\mathrm{SO}} / \phi_{0}<3$ compatible with previous studies. ${ }^{4,12,21}$ Equations (1)-(3) result in the energy splitting $\Delta_{\text {SO }}$ at $B_{\|}=0$ (assuming $k_{\|}=0$ ),

$$
\Delta_{\mathrm{SO}}=\frac{4 \hbar v_{F}}{d} \frac{\phi_{\mathrm{SO}}}{\phi_{0}} \approx 2.5 \pm 0.8 \mathrm{meV} .
$$

This value corresponds to $\sim 30 \mathrm{~K}$ and explains the disappearance of the double-peak structure and the single conductance maximum at $B_{\|}=B_{0} \simeq B_{0}^{*}$ for the $82 \mathrm{~K}$ trace of Fig. 1 . Because $\Delta_{\text {SO }}$ is inversely proportional to the diameter, it becomes large for small-diameter tubes. ${ }^{23}$

With further increase in $\phi_{\mathrm{AB}}$, the energy gap $E_{g}$ linearly opens again as both orbital subbands gradually move away from the corner points of the Brillouin zone. The exponential decrease in $G$ at high fields [the inset of Fig. 1(a)] is thus explained by charge carriers thermally activated over the magnetic-field-induced band gap, as described by previous authors. ${ }^{5,24}$ Since the inset in Fig. 1(a) suggests that the conductance depends exponentially on the band gaps $E_{g}^{0}$ and $E_{g}\left(B_{0}^{*}\right)$, they dominate the conductance at $B_{\|}=0$ and $B_{\|}=B_{0}^{*}$. Hence, the approximate equality $G(0) \approx G\left(B_{0}^{*}\right)$ inferred from Fig. 1(a) suggests the following relation between the two energy scales $E_{\text {curv }}$ and $\Delta_{\mathrm{SO}}$ :

TABLE I. Determination of the chirality from $G\left(B_{\|}\right)$at the charge-neutrality point. $\phi_{\text {SO }}$ was calculated from Eq. (3) with the measured value of $\Delta B=8 \mathrm{~T}$ and used as input for the evaluation of $\Delta_{\text {SO }}$ using Eq. (4).

\begin{tabular}{ccccccc}
\hline \hline & $\begin{array}{c}d \\
(n, m)\end{array}$ & $\begin{array}{c}\theta \\
(\mathrm{nm})\end{array}$ & $\begin{array}{c}E_{\text {curv }} \\
(\mathrm{meV})\end{array}$ & $\begin{array}{c}B_{0}, B_{0}^{*} \\
(\mathrm{~T})\end{array}$ & $\begin{array}{c}\phi_{\mathrm{SO}} \\
\left(10^{-3} \phi_{0}\right)\end{array}$ & $\begin{array}{c}\Delta_{\mathrm{SO}} \\
(\mathrm{meV})\end{array}$ \\
\hline$(12,9)$ & 1.43 & 25.3 & 4.6 & 7.8 & 1.54 & 2.36 \\
$(13,10)$ & 1.56 & 25.7 & 3.5 & 5.5 & 1.84 & 2.58 \\
$(16,10)$ & 1.78 & 22.4 & 4.7 & 6.4 & 2.39 & 2.94 \\
$(18,9)$ & 1.86 & 19.1 & 6.0 & 7.8 & 2.63 & 3.08 \\
$(19,10)$ & 2.00 & 19.8 & 4.9 & 5.9 & 3.02 & 3.30 \\
$(12,3)$ & 1.08 & 10.9 & 28.1 & 63.1 & 0.88 & 1.78 \\
$(17,2)$ & 1.42 & 5.5 & 18.5 & 31.6 & 1.51 & 2.33 \\
\hline \hline
\end{tabular}




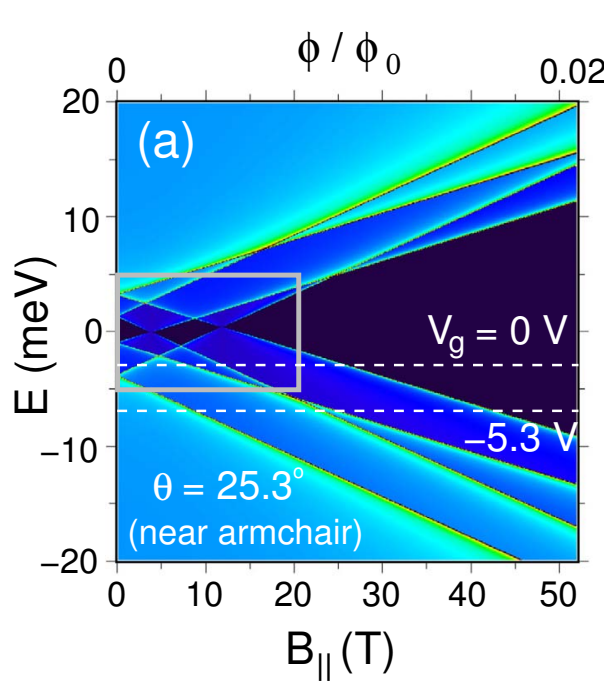

$(12,9)$

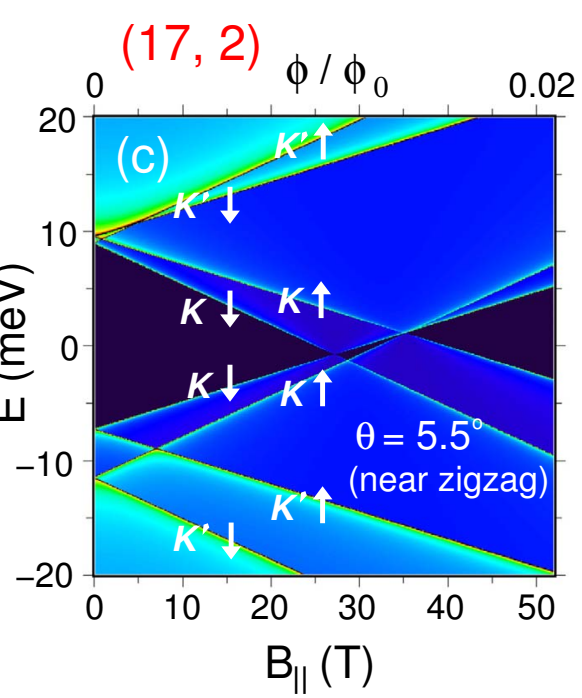

FIG. 2. (Color online) (a) Calculated density of states in a parallel magnetic field for the (12,9) CNT. (b) Zoom into the area bounded by the gray box clearly shows that the band gap is closed at $B_{1}$ and $B_{2}$ in good agreement with the peak positions observed in Fig. 1(a). White arrows indicate the spin polarization of the bands near the crossing points $B_{1}$ and $B_{2}$. (c) DOS calculated for the (17,2) CNT. Due to the larger curvature-induced band gap of the $(17,2)$ tube with $\theta$ close to $0^{\circ}$, the Zeeman-energy splitting at $B_{0}\left(g \mu_{B} B_{0} \approx 3.7 \mathrm{meV}\right)$ is larger than the spin-orbit energy splitting $\left(\Delta_{\mathrm{SO}} \approx 2.33 \mathrm{meV}\right)$. Hence, the SOI-induced peak splitting is pronounced for the CNTs with chiral angles close to $30^{\circ}$. Orbital ( $K$ and $\left.K^{\prime}\right)$ and spin states (white arrows) are indicated.

$$
\frac{E_{g}^{0}}{E_{g}\left(B_{0}^{*}\right)}=\frac{E_{\mathrm{curv}}-\Delta_{\mathrm{SO}}}{\Delta_{\mathrm{SO}}} \approx 1
$$

We now turn to the discussion of the effect of tube chirality. The strong dependence of $B_{0}$ and $B_{0}^{*}$ on the chirality can be used to identify the chiral indices of small-band-gap CNTs. $^{5}$ Out of 53 small-band-gap CNTs with $d$ $=1.5 \pm 0.5 \mathrm{~nm}$, only five tubes $[(12,9),(13,10),(16,10)$, $(18,9)$, and $(19,10)]$ display values of $B_{0} \approx 5-8 \mathrm{~T}$ compatible with our data while $B_{0}$ can take much larger values for other CNTs, e.g., the $(12,3)$ and $(17,2)$ tubes.

Table I lists values of $\phi_{\mathrm{SO}}$ and $\Delta_{\mathrm{SO}}$ for these chiralities, calculated from Eqs. (3) and (4) and the observed $\Delta B=8 \mathrm{~T}$. The $\phi_{\mathrm{SO}}$ of the $(12,9)$ tube is closest to $\phi_{\mathrm{SO}} \approx 10^{-3} \phi_{0}$, predicted in Ref. 12 and measured in Ref. 4. When we further take into account the condition $E_{\text {curv }} \approx 2 \Delta_{\text {So }}$ for the CNT measured [Eq. (5)], we realize that the $(12,9)$ and $(18,9)$ tubes satisfy this constraint best.

Taking the $(12,9)$ tube with the chiral angle $\theta=25.3^{\circ}$ (close to the armchair configuration) as the most probable candidate, we calculated the density of states (DOS) in a parallel magnetic field. We used the periodic boundary conditions, which are suitable for very long nanotubes. In shorter CNTs the open boundary condition in the axial direction gives rise additionally to edge effects, which are, however, beyond the scope of this work. For comparison, we show the DOS of a $(17,2)$ tube, which has almost the same diameter but a very different chiral angle $\theta=5.5^{\circ}$ close to the zigzag configuration.

From Fig. 2, it becomes apparent that in an applied magnetic field the band edges change with four distinct slopes away from the two Kramers doublets both in the electron and hole bands, reflecting the orbital and Zeeman splitting. The DOS calculated for the $(12,9)$ tube explains the evolution of the magnetoconductance very well. The band gap is closed at
$B_{1}$ by the spin down and subsequently at $B_{2}$ by the spin-up subband, in very good agreement with the observed double peaks at the CNP. The calculated energy gaps at zero field and at $B_{0}^{*}$ agree with Eqs. (4) and (5). On the other hand, the DOS calculated for the $(17,2)$ CNT predicts a significantly reduced intermediate gap region in Fig. 2(c), in spite of almost the same $d$ and $\Delta_{\text {So }}$, when compared with the $(12,9)$ tube. The $(17,2)$ tube has a much larger curvature-induced gap $E_{\text {curv }} \approx 18.5 \mathrm{meV}$, resulting in a much higher $B_{0}$

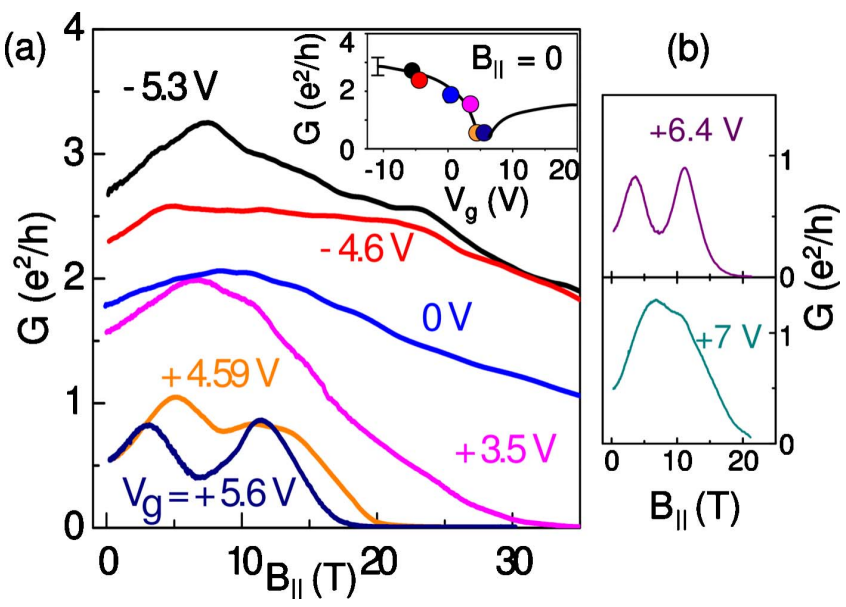

FIG. 3. (Color online) (a) $G\left(B_{\|}\right)$traces at $4.2 \mathrm{~K}$ for the hole side of the CNP. As the band gap grows with $B_{\|}$at high fields, the gate characteristic $G\left(V_{g}\right)$ exhibits the behavior of a $p$-type CNT fieldeffect transistor with on-off conductance ratio of several orders of magnitude. The inset shows $G\left(V_{g}\right)$ at $B_{\|}=0$. The dots correspond to the traces of $G\left(B_{\|}\right)$in main figure. The black solid line in the inset is a fit to experimental points (see error bar) given as a guideline to the eyes. (b) $G\left(B_{\|}\right)$curves for the electron side of the CNP. The double-peak structure is observed only when the Fermi energy is tuned close to the CNP at $V_{g} \simeq+6 \mathrm{~V}$. 
$\approx 31.6 \mathrm{~T}$. Because the spin-orbit gap competes with the Zeeman splitting, the peak splitting in $G\left(B_{\|}\right)$is most pronounced for near armchair tubes with chiral angles close to $30^{\circ}$.

So far, all our discussion focused on the vicinity of the charge-neutrality point. As a crucial test of our analysis, we traced the evolution of the $G\left(B_{\|}\right)$curves for various values of gate voltage. In Fig. 3(a), magnetoconductance traces at 4.2 $\mathrm{K}$ are displayed for the hole side of the CNP. Deeply inside the hole band, the conductance exceeds $3 e^{2} / h$, close to the theoretical limit of $4 e^{2} / h$. This shows that our device is in the ballistic regime, where the conductance is determined by the number of available subbands with an average transmission probability of $\sim 0.8$. At $B_{\|}=0$, the hole conductance is around $3 e^{2} / h$ and diminishes down to $\sim 0.5 e^{2} / h$ as $E_{F}$ is tuned toward the CNP $\left(V_{g}^{*} \sim+6 \mathrm{~V}\right)$. While the magnetoconductance is initially positive at low fields, it becomes negative at high fields $\left(B_{\|} \gg B_{0}\right)$ for all gate voltages, indicating the growth of $E_{g}$ due to the $\mathrm{AB}$ effect. At $B_{\|}>30 \mathrm{~T}$, the gate characteristic $G\left(V_{g}\right)$ exhibits the behavior of a $p$-type CNT field-effect transistor with an on-off conductance ratio $>10^{3}$. The double-peak structure is pronounced only in the vicinity of the CNP $\left(+5.6 \leq V_{g}^{*} \leq+6.4 \mathrm{~V}\right)$. Two additional $G\left(B_{\|}\right)$ curves, presented in Fig. 3(b), show that the two peaks merge again into one as $E_{F}$ is shifted to the electron side across the CNP.

Approximating the backgate coupling to the Fermi-energy shift as $\Delta E_{F} \approx 0.7 \times 10^{-3} e \Delta V_{g}$, the DOS calculation matches the gate voltages in Fig. 3. For instance, the conductance kinks observed at 8 and $24 \mathrm{~T}$ for the $G\left(B_{\|}\right)$curve at $V_{g}=$ $-5.3 \mathrm{~V}$ in Fig. 3(a), may be explained by the subsequent loss of $K_{\downarrow}^{\prime}$ subbands at $B \simeq 8 \mathrm{~T}$ and $K_{\uparrow}^{\prime}, K_{\downarrow}$ at $\simeq 24 \mathrm{~T}$ in the DOS. However, as the calculation neglects quantum interference effects in the Fabry-Perot regime, such as the AB beating effect, ${ }^{25}$ we cannot expect to explain all features of the measured magnetoconductance within our simple model.

In conclusion, we have investigated single-walled carbon nanotubes up to very high magnetic fields. The magnetoconductance of a quasimetallic tube shows a peculiar double peak, which can be explained in terms of spin-split conduction bands, separated by a strong spin-orbit interaction, which exceeds the Zeeman splitting. Our finding may open the path toward the application of CNTs as highly efficient ballistic spin filters.

This research was funded by the Deutsche Forschungsgemeinschaft within GRK 1570 and SFB 689 and partially supported by EuroMagNET under the EU Contract No. 228043.

\footnotetext{
*christoph.strunk@physik.uni-regensburg.de

${ }^{1}$ I. Žutić, J. Fabian, and S. D. Sarma, Rev. Mod. Phys. 76, 323 (2004).

${ }^{2}$ Y. K. Kato, R. C. Myers, A. C. Gossard, and D. D. Awschalom, Science 306, 1910 (2004).

${ }^{3}$ J. Wunderlich, B. Kaestner, J. Sinova, and T. Jungwirth, Phys. Rev. Lett. 94, 047204 (2005).

${ }^{4}$ F. Kuemmeth, S. Ilani, D. C. Ralph, and P. L. McEuen, Nature (London) 452, 448 (2008).

${ }^{5}$ G. Fedorov, A. Tselev, D. Jiménez, S. Latil, N. G. Kalugin, P. Barbara, D. Smirnov, and S. Roche, Nano Lett. 7, 960 (2007).

${ }^{6}$ T. Nakanishi and T. Ando, J. Phys. Soc. Jpn. 74, 3027 (2005).

${ }^{7}$ J. Kong, H. T. Soh, A. M. Cassell, C. F. Quate, and H. Dai, Nature (London) 395, 878 (1998).

${ }^{8}$ E. D. Minot, Y. Yaish, V. Sazonova, J.-Y. Park, M. Brink, and P. L. McEuen, Phys. Rev. Lett. 90, 156401 (2003).

${ }^{9}$ See supplementary material at http://link.aps.org/supplemental/ 10.1103/PhysRevB.82.041404 for experimental details and effects of the electron-hole asymmetric spin-orbit splitting in energy.

${ }^{10}$ H. Ajiki and T. Ando, J. Phys. Soc. Jpn. 62, 1255 (1993).

${ }^{11}$ See, e.g., J.-C. Charlier, X. Blase, and S. Roche, Rev. Mod. Phys. 79, 677 (2007), and references therein.

${ }^{12}$ T. Ando, J. Phys. Soc. Jpn. 69, 1757 (2000).

${ }^{13}$ D. V. Bulaev, B. Trauzettel, and D. Loss, Phys. Rev. B 77,
}

235301 (2008).

${ }^{14}$ W. Izumida, K. Sato, and R. Saito, J. Phys. Soc. Jpn. 78, 074707 (2009).

${ }^{15}$ J.-S. Jeong and H.-W. Lee, Phys. Rev. B 80, 075409 (2009).

${ }^{16} \mathrm{~S}$. Zaric, G. N. Ostojic, J. Kono, J. Shaver, V. C. Moore, M. S. Strano, R. H. Hauge, R. E. Smalley, and X. Wei, Science 304, 1129 (2004).

${ }^{17}$ U. C. Coskun, T.-C. Wei, S. Vishveshwara, P. M. Goldbart, and A. Bezryadin, Science 304, 1132 (2004).

${ }^{18}$ B. Lassagne, J.-P. Cleuziou, S. Nanot, W. Escoffier, R. Avriller, S. Roche, L. Forró, B. Raquet, and J.-M. Broto, Phys. Rev. Lett. 98, 176802 (2007).

${ }^{19}$ C. L. Kane and E. J. Mele, Phys. Rev. Lett. 78, 1932 (1997).

${ }^{20}$ A. Kleiner and S. Eggert, Phys. Rev. B 64, 113402 (2001).

${ }^{21}$ D. Huertas-Hernando, F. Guinea, and A. Brataas, Phys. Rev. B 74, 155426 (2006).

${ }^{22}$ L. Chico, M. P. Lopez-Sanchó, and M. C. Muñoz, Phys. Rev. B 79, 235423 (2009).

${ }^{23}$ Therefore, $\Delta_{\text {SO }}$ is larger for our nanotube, compared to the value observed for the tube $(d \sim 5 \mathrm{~nm})$ in Ref. 4.

${ }^{24}$ E. D. Minot, Y. Yaish, V. Sazonova, and P. L. McEuen, Nature (London) 428, 536 (2004).

${ }^{25}$ J. Cao, Q. Wang, M. Rolandi, and H. Dai, Phys. Rev. Lett. 93, 216803 (2004). 